\title{
Two-zone model for the broadband Crab nebula spectrum: mi- croscopic interpretation
}

\author{
F. Fraschetti ${ }^{1, a}$ and M. Pohl ${ }^{2,3}$ \\ ${ }^{1}$ Depts. of Planetary Sciences and Astronomy, University of Arizona, Tucson, AZ, 85721, USA \\ ${ }^{2}$ DESY, 15738 Zeuthen, Germany \\ ${ }^{3}$ Institute of Physics and Astronomy, University of Potsdam, 14476 Potsdam, Germany
}

\begin{abstract}
We develop a simple two-zone interpretation of the broadband baseline Crab nebula spectrum between $10^{-5} \mathrm{eV}$ and $\sim 100 \mathrm{TeV}$ by using two distinct log-parabola energetic electrons distributions. We determine analytically the very-high energy photon spectrum as originated by inverse-Compton scattering of the far-infrared soft ambient photons within the nebula off a first population of electrons energized at the nebula termination shock. The broad and flat $200 \mathrm{GeV}$ peak jointly observed by Fermi/LAT and MAGIC is naturally reproduced. The synchrotron radiation from a second energetic electron population explains the spectrum from the radio range up to $\sim 10 \mathrm{keV}$. We infer from observations the energy dependence of the microscopic probability of remaining in proximity of the shock of the accelerating electrons.
\end{abstract}

\section{Introduction}

The steady-state gamma-ray emission of the Crab Nebula has been observed in the past decade by a number of ground-based observatories, i.e., HEGRA [1], MAGIC [2], HESS II [3] and VERITAS [4]. The photon differential spectrum as determined by such experiments is consistent with a log-parabola distribution from $\sim 50 \mathrm{GeV}$ up to $100 \mathrm{TeV}$. We propose a two-zone model with two distinct populations of energetic electrons having two distinct log-parabola distributions injected at the same energy to explain the broadband spectrum from $10^{-5} \mathrm{eV}$ up to $100 \mathrm{TeV}$; however, the $\mathrm{MeV}$ region exceeds our prediction. In the literature, the broad and flat VHE peak at $200 \mathrm{GeV}$ joining the TeV-band spectrum observed with MAGIC $(0.05-30 \mathrm{TeV})$ with the GeV-band spectrum obtained with Fermi/LAT $(1-200 \mathrm{GeV})$ has been empirically modelled with a modified log-parabola with an additional freeparameter that is not physically justified [2]. We show that such VHE peak is naturally explained by inverse-Compton scattering off monochromatic Infra-Red ambient photons within the nebula of a log-parabola distribution of electrons. Synchrotron radiation explains the radio/ $X$-ray spectrum.

\section{Outline of the model for steady injection of energetic particles}

We assume that the number of electrons accelerated at the nebula termination shock (TS) with Lorentz factor $>\gamma$ is given by a log-parabola

$$
N(>\gamma)=N_{0}\left(\gamma / \gamma_{0}\right)^{-\left[s-1+r \log \left(\gamma / \gamma_{0}\right)\right]}
$$

\footnotetext{
a e-mail: ffrasche@1pl.arizona.edu
} 
where $N_{0}$ is a normalization constant, $\gamma_{0}$ is the electron injection Lorentz factor and the spectral parameters $s$ and $r$ are related to the microscopic statistical parameters.

\subsection{Inverse Compton flux}

The target photon population within the nebula is assumed to be isotropic in the frame of the plasma flowing downstream of the shock at the scale of the VHE electrons mean-free path. The cold photons are IC scattered by the isotropic energetic electrons.

For a photon ambient population with a phase space photon density (number of photons per unit of volume and energy) given by $n(\varepsilon)$ in the plasma frame, the spectrum of the scattered photons with initial energy between $\varepsilon$ and $\varepsilon+d \varepsilon$ and final energy $\varepsilon_{1}$ per single electron is [5]

$$
\frac{d N_{\gamma, e}}{d t d \varepsilon_{1}}=\frac{3 \sigma_{T} c}{4 \gamma^{2}} \frac{n(\varepsilon)}{\varepsilon} d \varepsilon G(q, \Gamma)
$$

where $\sigma_{T}$ is the Thomson cross-section, $c$ the speed of light in vacuum and

$$
G(q, \Gamma)=2 q \ln q+(1+2 q)(1-q)+\frac{\Gamma^{2} q^{2}(1-q)}{2(1+\Gamma q)}, \quad \Gamma=\frac{4 \varepsilon \gamma}{m_{e} c^{2}}, \quad q=\frac{\varepsilon_{1}}{\Gamma\left(m_{e} \gamma c^{2}-\varepsilon_{1}\right)} .
$$

and where Eqs $(2,3)$ are derived under the only restriction $\gamma \gg 1$. The calculation of the IC power emitted by a single electron in the plasma frame over the target photon distribution $n(\varepsilon)$ requires integration over the kinematically permitted photon final energies:

$$
P_{I C}=\int_{0}^{\infty} d \varepsilon \int_{0}^{\varepsilon_{1}^{M}} d \varepsilon_{1} \frac{d N_{\gamma, e}}{d t d \varepsilon_{1}} \varepsilon_{1}=12 \sigma_{T} c \gamma^{2} \int_{0}^{\infty} d \varepsilon \varepsilon n(\varepsilon) J(\Gamma),
$$

where $\varepsilon_{1}^{M}=\Gamma m_{e} \gamma c^{2} /(1+\Gamma)$ and $J(\Gamma)=\int_{0}^{1} d q q G(q, \Gamma) /(1+q \Gamma)^{3}$. The factor $J(\Gamma)$ can be approximated as follows: $J(\Gamma) \simeq 1 / 9$ in the Thomson limit $(\Gamma \ll 1)$ and $J(\Gamma) \simeq[\ln \Gamma-11 / 6] / 2 \Gamma^{2}$ in the extreme Klein-Nishima limit $(\Gamma \gg 1)[6]$.

We use the simplifying assumption of a monochromatic target photon field, $n(\varepsilon)=n_{0} \delta\left(\varepsilon-\varepsilon_{0}\right)$, where $\varepsilon_{0}$ is determined by the joint fit of Fermi/LAT and MAGIC data. Thus, the power $P_{I C}$ is given in the extreme Klein-Nishima limit by

$$
P_{I C}=\frac{3}{8} \sigma_{T} c\left(m c^{2}\right)^{2} \int_{0}^{\infty} d \varepsilon \frac{n(\varepsilon)}{\varepsilon}\left[\ln \frac{4 \varepsilon \gamma}{m_{e} c^{2}}-\frac{11}{6}\right] \simeq \frac{3}{8} \sigma_{T} c\left(m c^{2}\right)^{2} \frac{n_{0}}{\varepsilon_{0}}\left[\ln \frac{4 \varepsilon_{0} \gamma}{m_{e} c^{2}}-\frac{11}{6}\right] .
$$

Given the differential electron distribution from Eq. 1,

$$
\frac{d N(\gamma)}{d \gamma}=\frac{N_{0}}{\gamma_{0}}\left|-s+1-2 r \log \left(\gamma / \gamma_{0}\right)\right|\left(\frac{\gamma}{\gamma_{0}}\right)^{-\left[s+r \log \left(\gamma / \gamma_{0}\right)\right]},
$$

the total IC power is found by folding $d N(\gamma) / d \gamma$ with $P_{I C}$ in Eq. 5, $P_{I C}^{t o t}(v)=\int d \gamma P_{I C} d N(\gamma) / d \gamma$. Finally, defining the total IC flux at Earth from a source at distance $d$ as $v F_{v}^{I C}=P_{I C}^{\text {tot }}(v) / 4 \pi d^{2}$ leads to

$$
v F_{I C, v}(\varepsilon)=\frac{\mathcal{A}^{\prime}\left(\varepsilon_{0}\right)}{4 \pi d^{2}}\left[\ln \frac{4 \varepsilon_{0} \varepsilon}{\left(m_{e} c^{2}\right)^{2}}-\frac{11}{6}\right] \frac{N_{0}}{\gamma_{0}}\left[-s+1-2 r \log \left(\varepsilon / \bar{\varepsilon}_{0}\right)\right]\left(\varepsilon / \bar{\varepsilon}_{0}\right)^{-\left[s-1+r \cdot \log \left(\varepsilon / \bar{\varepsilon}_{0}\right)\right]}
$$

where we have defined $\bar{\varepsilon}_{0}=h \bar{\nu}_{0}=m_{e} c^{2} \gamma_{0}$ and $\mathcal{A}^{\prime}\left(\varepsilon_{0}\right)=(3 / 8)\left(h / m_{e} c\right) \sigma_{T}\left(m_{e} c^{2}\right)^{3} n_{0} \gamma_{0} / h \varepsilon_{0}$ and we have used the monochromatic approximation $h v \simeq m_{e} c^{2} \gamma$ for the extreme Klein-Nishima limit. Equation 7 is a log-parabola distribution multiplied by two logarithmically dependent pre-factors in the squared brackets of the right hand side. 

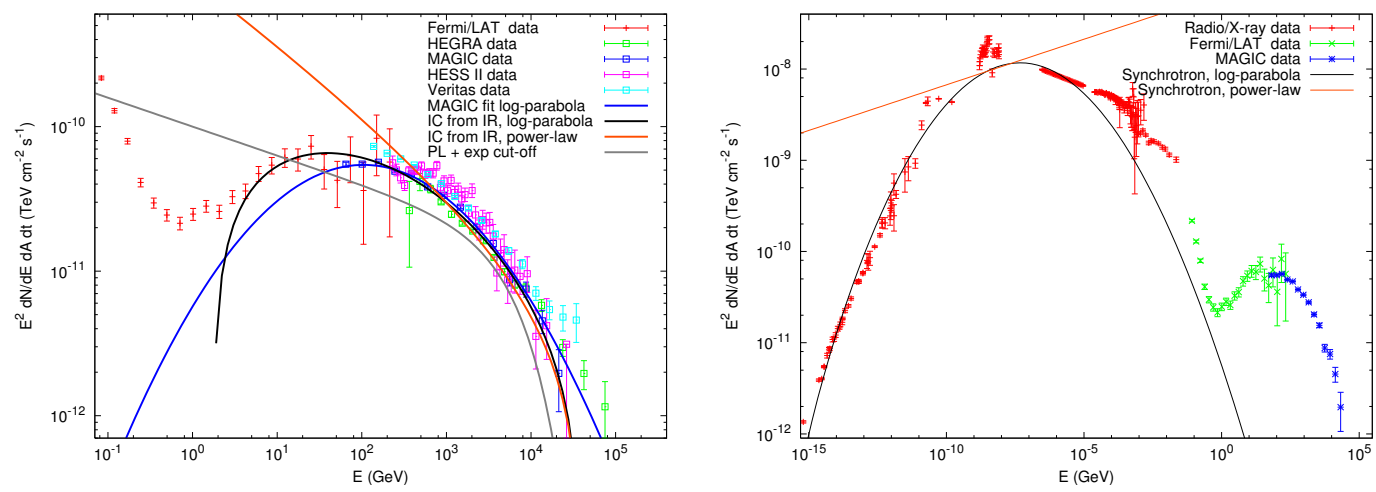

Figure 1. Left Theoretical photon differential spectrum (black) in Eq. 7 from inverse-Compton off a $\varepsilon_{0}=10^{-2}$ $\mathrm{eV}$ monochromatic target photon field. The theoretical curve with best-fit parameters (see Table 1) is compared with the data from Fermi/LAT [7], HEGRA [1], MAGIC [2], HESS II [3] and VERITAS [4] and the log-parabola fit (blue) of the MAGIC data with indexes $\alpha=2.47$ and $\beta=-0.24$ (see Eq. 9). The red-curve shows the same spectrum as the black curve with $r=0$. A power-law with exponential cut-off (grey) with slope 2.2 and energy cut-off $E_{c}=6 \mathrm{TeV}$ is reported for illustrative purpose. Right Theoretical photon differential spectrum (in black) from Eq. 8 with best-fit parameters (see Table 1). The red-curve shows the same spectrum as the black curve with $r=0$. The radio and soft $X$-rays data from ISO-SCUBA, Spitzer, XMM-Newton, SPI, IBIS/ISGRI (same set used for the broadband spectrum in [2]) are shown in red. Fermi/LAT and MAGIC data are overlaid for comparison.

\subsection{Synchrotron flux}

The synchrotron power emitted by a single electron, averaged over an isotropic electron distribution, is given in the plasma frame by $P_{s y n c}=\frac{\sigma_{T} c}{6 \pi} \gamma^{2} B^{2}$, where $B$ is the external magnetic field assumed to be uniform. The total synchrotron power is found by folding the single electron power with the differential electron spectrum in Eq. 6: $P_{\text {sync }}^{t o t}=\int d \gamma P_{\text {sync }} d N(\gamma) / d \gamma$. We use the monochromatic approximation with a characteristic synchrotron frequency $v_{s}=0.29\left(3 e \gamma^{2} B\right) /\left(4 \pi m_{e} c\right)=\gamma^{2} v_{s}^{0}$, so we recast $P_{\text {sync }}^{\text {tot }}=\int d \gamma P_{\text {sync }} d N(\gamma) /\left.d \gamma\right|_{v=v_{s}}$. Thus, for $v \simeq v_{s}$, the total flux at Earth $v F_{v}^{s y n c}$, related to synchrotron power $v F_{v}^{\text {sync }}=P_{\text {sync }}^{\text {tot }}(v) / 4 \pi d^{2}$ can be approximated as

$$
v F_{v}^{\text {sync }}(\varepsilon)=\frac{\sigma_{T} c}{12 \pi 4 \pi d^{2}} N_{0} B^{2} \gamma_{0}^{2}\left(\frac{\varepsilon}{\varepsilon_{s}^{0} \gamma_{0}^{2}}\right)^{-\frac{1}{2}\left[s-3+\frac{r}{2} \log \left(\varepsilon / \varepsilon_{s}^{0} \gamma_{0}^{2}\right)\right]}\left|-s+1-r \log \left(\varepsilon / \varepsilon_{s}^{0} \gamma_{0}^{2}\right)\right|
$$

where we have defined $\varepsilon_{s}^{0}=h v_{s}^{0}$.

\section{Comparison with broadband observations}

\subsection{GeV and multi-TeV spectrum}

We assume that energetic electrons inverse-Compton scatter off a gas of monochromatic IR photons within the nebula with energy $\varepsilon_{0}=10^{-2} \mathrm{eV}$ and number density $n_{0}=5 \times 10^{2} \mathrm{~cm}^{-3}$ (the distance from the source is $d=1.9 \mathrm{kpc}$ ). Figure 1, Left panel, compares the resulting analytic photon differential spectrum in the VHE range given by Eq. 7 with the spectra from various ground-based VHE observatories above $10 \mathrm{GeV}$ : HEGRA (300 GeV- $100 \mathrm{TeV}$ ) [1], MAGIC (50 GeV - $30 \mathrm{TeV}$ ) [2], HESS II 
(230 GeV - $25 \mathrm{TeV})$ [3] and VERITAS (115 GeV - $42 \mathrm{TeV})$ [4]. The best-fit parameters in Fig. 1 of the joint Fermi-LAT and MAGIC data are reported in Table 1. In Fig. 1, Left panel, the overlaid $\log$-parabola empirical fit of the solely MAGIC data provided by [2] has the form, modulo an $\varepsilon^{2}$ factor,

$$
\frac{d \mathcal{N}}{d \varepsilon d A d t}(\varepsilon)=f_{0}\left(\varepsilon / \varepsilon_{0}^{M}\right)^{-\alpha+\beta \cdot \log \left(\varepsilon / \varepsilon_{0}^{M}\right)}
$$

with power-law index $\alpha=2.47 \pm 0.01$, curvature $\beta=-0.24 \pm 0.01$, scale $\varepsilon_{0}^{M}=1 \mathrm{TeV}$ (note that $\varepsilon_{0}^{M}=\bar{\varepsilon}_{0}$ ) and normalization factor $f_{0}=3.23 \times 10^{-11} \mathrm{TeV}^{-1} \mathrm{~cm}^{-2} \mathrm{~s}^{-1}$. We note that, not suprisingly, the best-fit value of $2-\alpha$ and $\beta$ of MAGIC data are inconsistent with our value of joint Fermi/LAT MAGIC fit $-(s-1)$ and $r$. The grey curve in Fig. 1 shows that a power-law with exponential cut-off is unable to reproduce the VHE spectrum from the peak up to the highest energies (here the cut-off is set to $E_{c}=6 \mathrm{TeV}$ from [2]). It turns out that there is no parameter combination for a simple power-law distribution of the electrons $(r=0)$ able to reproduce the broadband observations in the assumption of monochromatic photon target gas, but a different distribution of energetic particles is needed.

Figure 1, Left panel, shows that the MAGIC empirical log-parabola in Eq. 9 and our solution in Eq. 7 equally well reproduce the MAGIC data down to the IC peak at $\simeq 200 \mathrm{GeV}$. Our theoretical curve is also able to reproduce the broadness of the IC peak and the high-energy Fermi/LAT data; the logarithmic pre-factor in the second squared parentheses in Eq. 7 governs the width of the GeV peak that a simple log-parabola curve fails to reproduce.

The simple theoretical curve derived here is in good agreement with both the Fermi/LAT rising data up to the IC peak and the VHE data. The declining part of the Fermi-LAT spectrum below $\simeq 1$ $\mathrm{GeV}$ is likely to arise from a distinct particle population or distinct acceleration process. The best-fit value of the VHE band in a magnetic field with strength $B=140 \mu \mathrm{G}$ gives a characteristic synchrotron photon energy $\varepsilon_{s}^{0} \gamma_{0}^{2}=2.7 \mathrm{eV}$. The corresponding $v F_{v}^{s y n c}=P_{\text {sync }}^{\text {tot }}(v) / 4 \pi d^{2}$ (see Eq.8) with parameters fixed by fit of IC spectrum is inconsistent with radio and $X$-ray data.

\subsection{Radio and X-ray spectrum}

By using a constant and uniform magnetic field with strength $B=140 \mu \mathrm{G}$, the radio and soft $X$-ray data $\left(10^{-5} \mathrm{eV}\right.$ up to $\left.\sim 10 \mathrm{keV}\right)$ can be reproduced with the steady synchrotron emission from a logparabola given by Eq. 8, as shown in Fig.1, right panel. However, the best-fit parameters differ from those in the VHE range (see Table 1) with the same injection $\gamma_{0} \simeq 2 \times 10^{6}$. Such a parameter set leads to a predicted IC flux inconsistent with VHE observations. In addition, Fig. 1 shows that the range $0.1-10^{3} \mathrm{MeV}$ requires a third energetic particles population or emission process that is not discussed in this work. The best-fit values of the indexes $s, r$ are about twice for synchrotron than for IC (see Table 1). The reason is in the monochromatic approximation leading to the difference of a factor $1 / 2$ in the exponential of the log-parabola in Eq. 8 as compared to Eq. 7: the characteristic frequency scales as $\gamma$ for IC and as $\gamma^{2}$ for synchrotron. We note that the Synchrotron self-Compton with the best-fit parameters of the synchrotron radiation is inconsistent with the Fermi/LAT and MAGIC data.

\section{Discussion and Conclusion}

The log-parabola particle spectrum can be derived in the assumption that the probability for energetic electrons of remaining in proximity of the acceleration region and not being advected downstream or leak upstream of the shock, $P$, decreases with $\gamma$ as $P=g / \gamma^{-q}$, with the normalization constant $g$ and $q>0$ [8]. As well known, a $P$ independent of energy results in a power-law particle spectrum. For a quasi-perpendicular shock geometry such as the nebula TS, to the lowest order in $U_{1} / v$ where 
Table 1. Best-fit value of the electron log-parabola distribution parameters within the specified range

\begin{tabular}{lllll}
\hline & $s$ & $r$ & $N_{0}\left(10^{44}\right)$ & $\bar{\varepsilon}_{0}=m_{e} c^{2} \gamma_{0}(\mathrm{TeV})$ \\
\hline Synchrotron & $2.795 \pm 0.003$ & $0.2523 \pm 0.0018$ & $10^{3} \pm 90$ & $1.049 \pm 99$ \\
$\left(5 \times 10^{-6} \mathrm{eV}-10 \mathrm{keV}\right)$ & & & & \\
$\mathrm{IC}$ & $1.3616 \pm 0.0054$ & $0.06557 \pm 0.0024$ & $1.000 \pm 0.009$ & $1.003 \pm 0.099$ \\
$\left(2.2-10^{4}\right) \mathrm{GeV}$ & & & & \\
\hline
\end{tabular}

$U_{1}$ is the upstream flow speed, we find $q=2 r U_{1} / c$ (see [9] for detailed discussion). If $U_{1} / c \simeq 0.2$, we find for the two electron populations $q \simeq 0.026$ (VHE) and $q \simeq 0.1$ (Radio/X-ray). The origin of the two distinct electron populations is not discussed in this short note.

\section{Acknowledgment}

We thank D. Mazin, N. Otte and M. Holler for sharing low-energy, VERITAS and HESS II data respectively. The work of FF was supported in part by NASA under grants NNX13AG10G and NNX15AJ71G. FF thanks the organisers of the $6^{\text {th }}$ RICAP held in 2016. FF thanks for the hospitality the Harvard/Smithsonian Center for Astrophysics where part of this work was performed.

\section{References}

[1] F. Aharonian, A. Akhperjanian, M. Beilicke, K. Bernlöhr, H.G. Börst, H. Bojahr, O. Bolz, T. Coarasa, J.L. Contreras, J. Cortina et al., ApJ614, 897 (2004), astro-ph/0407118

[2] J. Aleksić, S. Ansoldi, L.A. Antonelli, P. Antoranz, A. Babic, P. Bangale, J.A. Barrio, J. Becerra González, W. Bednarek, E. Bernardini et al., Journal of High Energy Astrophysics 5, 30 (2015), 1406.6892

[3] M. Holler, D. Berge, C. van Eldik, J.P. Lenain, V. Marandon, T. Murach, M. de Naurois, R.D. Parsons, H. Prokoph, D. Zaborov et al., ArXiv e-prints (2015), 1509.02902

[4] Meagher, K. for the VERITAS Collaboration, ArXiv e-prints (2015), 1508.06442

[5] G.R. Blumenthal, R.J. Gould, Reviews of Modern Physics 42, 237 (1970)

[6] R. Schlickeiser, MNRAS398, 1483 (2009)

[7] R. Buehler, J.D. Scargle, R.D. Blandford, L. Baldini, M.G. Baring, A. Belfiore, E. Charles, J. Chiang, F. D'Ammando, C.D. Dermer et al., ApJ749, 26 (2012), 1112 . 1979

[8] E. Massaro, M. Perri, P. Giommi, R. Nesci, AEA413, 489 (2004), astro-ph/0312260

[9] F. Fraschetti, M. Pohl, submitted, ArXiv e-prints (2017), 1702 .00816 